\title{
Institution, événement et histoire chez Merleau-Ponty
}

Par ROBERTO TERZI

Archives Husserl de Paris (ENS/CNRS)

Résumé Nous examinons ici les différentes implications du concept d'institution de Merleau-Ponty pour une phénoménologie de l'historicité. Après avoir rappelé des débats concernant le " retour de l'événement » dans l'histoire contemporaine, et l'absence de ce thème dans les phénoménologies françaises de l'événement, nous essayons de montrer en un premier moment la possible contribution des analyses de Merleau-Ponty à cette question : son concept d'institution implique, en effet, aussi une réflexion sur l'événement, qui s'articule sur plusieurs plans et peut être utilement rapprochée de la conception de l'événement qui s'est diffusée dans l'historiographie contem-poraine. La portée des réflexions de Merleau-Ponty ne se limite bien sûr pas à une phénoménologie de l'événement historique, mais implique une compréhension d'ensemble de l'historicité, qui doit être pensée dans la conjonction de l'événement et de la fécondité de la tradition, en deçà des oppositions entre logique et contingence, subjectif et objectif, activité et passivité. Cet approfondissement permettra d'aborder aussi les concepts d'histoire sauvage et histoire verticale, ainsi que l'idée programmatique d'une " métaphysique de l'histoire », dont nous essayons de donner une interprétation à plusieurs niveaux. Précisément ce dernier thème nous conduira enfin à soulever des questions critiques concernant la subordination des thèmes de l'altérité, de la différence, de la nouveauté dans la compréhension merleaupontienne de l'histoire et du rapport entre les cultures. 


\section{1. Événement historique, phénoménologie, théorie de l'historiographie}

Dans un riche article de $2004^{1}$, Marlène Zarader examinait la large diffusion de ce «mot d'époque » qu'est l'événement, aussi bien dans la phénoménologie française contemporaine (en particulier dans les œuvres de Marion et Romano, mais aussi dans certains textes de Benoist et Dastur) que dans l'historiographie la plus récente. C'est précisément ce parallèle qui permettait à Zarader de relever une limite des phénoménologies françaises de l'événement, à savoir l'absence d'une thématisation de l'événement historique, malgré l'insistance générale sur la question de l'événement comme paradigme d'une nouvelle phénoménologie ; absence qui va avec une référence assez stéréotypée à la conception historienne de l'événement comme fait positif, atomique, objectif, à son tour inséré dans une vision encore linéaire et continuiste de l'histoire ${ }^{2}$. Référence stéréotypée et datée, car, comme Zarader le montre, la théorie et la pratique historiennes ne sont pas restées à cette vision, mais, au cours des dernières décennies, elles ont connu un véritable «retour de l'événement» qui a impliqué une analyse renouvelée et beaucoup plus complexe de celui-ci ${ }^{3}$. "Retour de l'événement» était en effet le titre d'un article, que l'on peut qualifier d'inaugural, publié par Pierre Nora en $1974^{4}$ : relevant le retour en puissance de l'événement dans l'histoire contemporaine (en l'occurrence à partir des faits de mai 1968), aussi par son entrelacement indissoluble avec les médias qui le diffusent, l'amplifient et même le produisent, Nora ouvrait en effet la voie à une nouvelle attention de la part de l'historiographie au thème de l'événement.

Cette attention n'a eu de cesse de croître dans les années suivantes et dans son ouvrage de 2010 La renaissance de l'événement ${ }^{5}$ l'historien François Dosse se propose ainsi non seulement de faire état de cette diffusion du thème, mais aussi d'expliciter et valoriser la conception de l'événement qu'elle implique; et si le texte développe un dialogue aussi avec plusieurs analyses philosophiques, où les références principielles semblent être Ricœur, Deleuze et Foucault, Dosse rappelle expressément et relance à son tour la critique de M. Zarader à l'égard des phénoménologies françaises de

\footnotetext{
${ }^{1}$ M. Zarader, «L'événement entre phénoménologie et histoire », Tijdschrift voor Filosofie, n. 2, 2004, p. 287-321.

${ }^{2}$ Ibid., p. 300-301, 304-305.

${ }^{3}$ Ibid., p. 302 sq.

${ }^{4}$ P. Nora, "Le retour de l'événement», in J. Le Goff \& P. Nora (éd.), Faire de l'histoire, vol. 1, Paris, Gallimard, 1974, p. 210-227.

${ }^{5} \mathrm{~F}$. Dosse, La renaissance de l'événement. Un défi pour l'historien : entre sphinx et phénix, Paris, PUF, 2010.
} 
l'événement, pour leur « oubli » à la fois du thème de l'événement historique et du renouvellement des conceptions historiennes ${ }^{1}$. Comme on sait, le rôle de l'événement avait été fortement limité par l'École des Annales, qui y voyait le symbole d'une historiographie attentive presque exclusivement à cette écume de l'histoire représentée justement par les événements politiques et militaires ; cette histoire " événementielle » devait alors être dépassée en faveur d'une analyse des structures économiques, sociales, culturelles, géographiques, caractérisées par la "longue durée». Or d'une part, selon François Dosse, certaines formes extrêmes de l'historiographie issue des Annales risquaient de basculer dans une anthropologie anhistorique, incapable de rendre compte du changement et donc difficilement compatible avec les préoccupations de l'histoire ${ }^{2}$; d'autre part, l'histoire contemporaine, aussi par les formes de sa diffusion, nous a rappelé qu'on ne saurait faire l'économie de l'événement, comme irruption contingente du nouveau, pour la compréhension de l'histoire. Mais, pour ainsi dire, l'événement qui fait alors retour n'est pas le «même » événement qui avait été critiqué auparavant : il ne s'agit pas de revenir en deçà de la critique de l'École des Annales vers une approche positiviste de l'événement comme fait ponctuel et objectif, mais d'élaborer une nouvelle conception de l'événement qui puisse rendre compte de son caractère pluridimensionnel, de la complexité de son rôle dans l'histoire, de son rapport avec l'opération historiographique elle-même. Nous nous limitons ici, à ce propos, à rappeler deux éléments fondamentaux. En premier lieu, comme déjà Paul Ricœur le remarquait dans un article qui portait le même titre que celui de $\mathrm{Nora}^{3}$, il s'agit de compliquer les rapports entre événement, conjoncture, structure : l'événement est une composante essentielle de l'ensemble solidaire qu'il forme avec la structure et la conjoncture ; non seulement il met en lumière, en les exhibant et en les condensant en soi, la conjoncture et la structure ${ }^{4}$, mais il peut aussi contribuer à les modifier en tant qu'événement créateur et sur-signifiant. En deuxième lieu, il ne s'agit pas d'envisager l'événement comme un fait positif et objectif dont il faudrait établir les causes dans la série d'un temps chronologique, mais

${ }^{1}$ Ibid., p. $95-98$.

${ }^{2}$ Ibid., p. 70-72.

${ }^{3}$ Cf. P. Ricœur, « Le retour de l'Événement », in Mélanges de l'École française de Rome. Italie et Méditerranée, T. 104, n. 1, 1992, p. 29-35.

${ }^{4}$ C'est pourquoi, de façon quelque peu paradoxale, J. Le Goff a pu affirmer que le retour de l'événement n'est pas une défaite, mais «le triomphe de la problématique des Annales »: J. Le Goff, "Les "retours" dans l'historiographie française actuelle ", Les Cahiers du Centre de Recherches Historiques, n. 22, 2009, en ligne à l'adresse https://ccrh.revues.org/2322, p. 4 de la version pdf. 
comme un événement signifiant, dont le sens réside dans les traces qu'il laisse et dans le champ d'effets qu'il ouvre. Autrement dit, et pour reprendre la formule efficace de F. Dosse, ce qui est en jeu c'est « un déplacement de l'approche de l'amont de l'événement vers son aval, de ses causes à ces traces », un "déplacement de l'événementialité vers sa trace et ses héritiers $\gg{ }^{1}$ : il s'agit par conséquent d'envisager l'événement du point de vue des effets qu'il provoque, du champ qu'il ouvre, mais aussi des reprises multiples (narratives, médiatiques, historiographiques) auxquelles il donne lieu et qui font donc partie, de plein droit, du sens de l'événement lui-même. Selon l'affirmation de Michel De Certeau, assumée par F. Dosse comme représentative de cette nouvelle conception, l'événement est «ce qu'il devient $»^{2}$.

Notre propos ici ne sera pas celui d'aborder directement l'ensemble de ce contexte, avec les références qui le constituent et les problèmes qui en découlent. Si nous avons esquissé rapidement ce cadre, c'est en un sens pour relancer l'interrogation de Marlène Zarader concernant le rapport entre phénoménologie et événement historique et pour proposer un rapprochement avec un autre moment, théorique et historique, de la phénoménologie. Il s'agit du concept d'institution élaboré par Merleau-Ponty, dont nous voudrions montrer qu'il permet, au moins sous un certain aspect, d'envisager de façon féconde la question de l'événement historique en phénoménologie, et cela dans une correspondance singulière précisément avec la conception de l'événement impliquée par son retour sur la scène de l'historiographie contemporaine ${ }^{3}$. Après avoir rappelé les traits généraux du concept d'institution, nous expliciterons son rapport avec le thème de l'événement et les aspects pour lesquels il se propose comme une voie pour réfléchir sur l'événement historique dans le cadre qui a constitué notre point de départ. Naturellement l'intention et la portée des analyses de Merleau-Ponty sont avant tout phénoménologiques et philosophiques (et, comme nous le verrons,

\footnotetext{
${ }^{1}$ F. Dosse, La renaissance de l'événement, op. cit., respectivement p. 1 et p. 176.

${ }^{2}$ M. De Certeau, «Prendre la parole» (1968), in Id., La prise de parole et autres écrits politiques, Paris, Points/Seuil, 1994, p. 51 ; cité par F. Dosse, La renaissance de l'événement, op. cit., p. 1, 167.

${ }^{3}$ Un des buts implicites de nos analyses est alors celui d'élargir le champ de la confrontation entre la théorie de l'historiographie et la philosophie française contemporaine : si les références privilégiées des historiens, à propos des questions rappelées, semblent être Ricœur d'une part, Foucault et Deleuze d'autre part, il nous semble que des rapprochements peuvent être explorés aussi avec Merleau-Ponty et avec la problématique de l'événement chez Derrida, que nous nous proposons d'aborder dans un autre texte.
} 
même «métaphysiques »), et il s'agira alors, dans un deuxième moment, d'esquisser la conception plus générale de l'historicité que l'on peut dégager des analyses sur l'institution. Cet élargissement nous permettra enfin d'indiquer aussi ce qui nous paraît être certaines limites de la position de Merleau-Ponty à propos de la compréhension de l'histoire et de son « événementialité ».

\section{Institution et événement historique chez Merleau-Ponty}

Le terme « institution » chez Merleau-Ponty est avant tout la traduction que le phénoménologue français adopte, à partir d'un certain moment, pour le concept husserlien de Stiftung, qui apparaît à plusieurs endroits des derniers textes de Husserl et en particulier dans le cadre de sa phénoménologie génétique. Stiftung (qui a été traduit aussi par «fondation» ou par « instauration $\gg)$ indique chez Husserl le premier acte par lequel la conscience acquiert et « fonde » un certain sens objectif, qui demeure ensuite comme un acquis stable et permanent dans son expérience; cette dynamique peut être retrouvée aussi bien au niveau de la formation de la conscience singulière et de ses habitus, qu'au niveau de l'historicité, où la Stiftung (plus précisément l'Urstiftung) indique alors l'institution originaire d'une formation de sens historique qui demeure ensuite disponible comme une tradition ${ }^{1}$. Chaque formation de sens se meut ainsi pour Husserl dans le renvoi circulaire entre une institution originaire (Urstiftung) dont les résultats se sédimentent, une ré-institution (Nachstiftung) qui réactive et relance le sens de l'institution originaire après son oubli dans la sédimentation, une institution finale (Endstiftung) comme pôle téléologique vers lequel toute formation de sens est orientée.

Merleau-Ponty a été très tôt sensible aux potentialités de ce concept husserlien, qu'il reprend et développe de façon originale surtout à partir du début des années 1950. Il convient aussi de remarquer l'importance de l'" effet de champ » que Merleau-Ponty obtient par la traduction d' " institution », au moins à deux égards : en premier lieu, « institution » joint en soi

\footnotetext{
${ }^{1}$ Nous avons abordé le concept de Stiftung/institution dans l'article «Événement, champ, trace : Le concept phénoménologique d'institution », Philosophie, 131/4, 2016, p. 52-68, auquel nous nous permettons de renvoyer aussi pour plus de détails sur les éléments husserliens rappelés. Parmi les textes principaux de Husserl, la référence est évidemment avant tout aux Méditations cartésiennes et à la Crise des sciences européennes.
} 
de façon efficace les significations de l'acte (ou de l'événement) d'instituer et du résultat de cet acte («institution » comme ce qui a été institué) — et, comme nous le verrons, cette duplicité de plans est précisément au cœur de l'élaboration merleaupontienne du concept; en deuxième lieu, et par là même, le terme « institution » permet de mettre en communication la problématique strictement phénoménologique et les multiples institutions de sens (anthropologiques, culturelles, politiques) qui peuplent les différents champs de notre expérience.

Un passage remarquable de Le langage indirect et les voix du silence (1952) nous indique le noyau essentiel du concept de Stiftung et la façon dont Merleau-Ponty le reprend à son compte (même si ici la traduction par « institution » n'a pas encore été fixée) :

Husserl a employé le beau mot de Stiftung, — fondation ou établissement, pour désigner d'abord la fécondité illimitée de chaque présent qui, justement parce qu'il est singulier et qu'il passe, ne pourra jamais cesser d'avoir été et donc d'être universellement, - mais surtout celle des produits de la culture qui continuent de valoir après leur apparition et ouvrent un champ de recherches où ils revivent perpétuellement. C'est ainsi que le monde dès qu'il l'a vu, ses premières tentatives de peintre et tout le passé de la peinture livrent au peintre une tradition, c'est-à-dire, commente Husserl, le pouvoir d'oublier les origines et de donner au passé, non pas une survie qui est la forme hypocrite de l'oubli, mais une nouvelle vie, qui est la forme noble de la mémoire ${ }^{1}$.

La Stiftung/institution indique donc la fécondité illimitée d'une singularité, de tout moment singulier de l'expérience qui ouvre un champ infini de reprises et de transformations. Et cette dynamique se trouve ici liée de façon privilégiée aux produits culturels et historiques, qui, institués une première fois, ouvrent le champ de leur propre tradition, laquelle implique à la fois et simultanément l'oubli de ses origines et la possibilité de connaître une vie toujours nouvelle dans les reprises auxquelles elle donnera lieu.

Cette «application» privilégiée aux domaines de la culture et de l'histoire n'exclut pas une extension généralisée du concept d'institution, que Merleau-Ponty entreprend en effet dans son cours au Collège de France de 1954-55, entièrement dédié à ce thème et intitulé L'institution dans l'histoire

\footnotetext{
${ }^{1}$ M. Merleau-Ponty, «Le langage indirect et les voix du silence », in Id., Signes, Paris, Gallimard, collection «Folio », 2001 (1960 pour l'édition originelle), p. 95, dorénavant cité LI.
} 
personnelle et publique ${ }^{1}$. Le concept d'institution est ici soumis à une généralisation qui le transforme en un nouveau pivot conceptuel, dont Merleau-Ponty s'attache à dégager le rôle à tous les niveaux de l'expérience, de la vie de l'organisme jusqu'aux champs de la culture et de l'histoire. Or il est remarquable que Merleau-Ponty, comme le résumé du cours le rappelle clairement, définisse ici l'institution par le thème de l'événement :

On entendait donc ici par institution ces événements d'une expérience qui la dotent de dimensions durables, par rapport auxquelles toute une série d'autres expériences auront sens, formeront une suite pensable ou une histoire, - ou encore les événements qui déposent en moi un sens, non pas à titre de survivance et de résidu, mais comme appel à une suite, exigence d'un avenir ${ }^{2}$.

Les institutions sont donc précisément des événements, qui dotent l'expérience de dimensions durables, ouvrent un champ dans lequel une série d'autres expériences pourront s'insérer et prendre sens ${ }^{3}$, et cela parce que ces dimensions durables ne sont pas quelque chose de statique et d'inerte, mais un sens en genèse qui fait appel à une suite, qui demande sa propre reprise et transformation.

Si Merleau-Ponty indique clairement sa dette par rapport à Husserl, il est en même temps évident qu'il transforme le concept d'institution à la lumière de sa propre position. Aussi le cours de 1954-1955 indique-t-il la prise de distance par rapport à Husserl et l'intention de développer le concept d'institution dans un sens non subjectiviste : "On cherche ici dans la notion d'institution un remède aux difficultés de la philosophie de la conscience. Devant la conscience, il n'y a que des objets constitués par elle», qui sont « le reflet exact des actes et des pouvoir de la conscience» et qui sont donc incapables de la relancer vers quelque chose d'autre que soi. Une philosophie

\footnotetext{
${ }^{1}$ Le cours est publié dans le volume M. Merleau-Ponty, L'institution/La passivité. Note de cours au Collège de France (1954-55), textes établis par D. Darmaillacq, C. Lefort et Stéphanie Ménasé, préface de C. Lefort, Paris, Belin, 2003, p. 31-154 ; dorénavant cité IP. Sur l'importance de ce cours dans l'itinéraire de Merleau-Ponty, cf. l'utile essai de R. Vallier, «Institution. The Significance of Merleau Ponty's 1954 course at the Collège de France », Chiasmi international, n. 7, 2005, p. 281-302.

${ }^{2}$ M. Merleau-Ponty, Résumés de cours. Collège de France, 1952-1960 (cours L'institution...), Paris, Gallimard, 1968, p. 61, nous soulignons ; dorénavant cité RC. ${ }^{3}$ Dans le cours Merleau-Ponty exprime ce point aussi en rapprochant l'institution de l'instauration de dimensions au sens cartésiens: "Donc institution [signifie] établissement dans une expérience (ou dans un appareil construit) de dimensions (au sens général, cartésien : système de référence) par rapport auxquelles toute une série d'autres expériences auront sens et formeront une suite, une histoire » (IP, p. 38).
} 
de la conscience est au fond incapable de rendre compte effectivement et concrètement du rapport du Je aux autres et à son passé. « Si le sujet était instituant, non constituant, on comprendrait au contraire qu'il ne soit pas instantané, et qu'autrui ne soit pas seulement le négatif de soi-même ${ }^{1}$. L'institution est donc un avènement pré-subjectif du sens, irréductible aux opérations d'une conscience et précisément pour cette raison fécond. Un avènement pré-subjectif et qui demeure asubjectif dans son développement : c'est pourquoi Merleau-Ponty peut écrire que «constituer en ce sens est presque le contraire d'instituer: l'institué a sens sans moi, le constitué n'a sens que pour moi et pour le moi de cet instant $»^{2}$. L'institution n'est donc pas la fixation d'une signification isolée, ni le simple reflet d'un acte de la conscience, mais, selon les termes fréquemment utilisés par Merleau-Ponty, l'ouverture d'un champ ou d'une dimension sur-individuelle, qui est l'Ineinander ou l'entre-deux des extases temporelles et des relations entre les sujets.

Ces définitions de Merleau-Ponty nous permettent de faire ressortir plus nettement le noyau de la fécondité théorique du concept d'institution et la possibilité de son rapprochement avec la problématique de l'événement historique que nous avons reconstruite au début. De ce point de vue le texte de Merleau-Ponty a pour nous une fonction exemplaire au double sens de l'expression : un exemple parmi d'autres d'une question théorique plus générale et un exemple paradigmatique pour son importance. La raison majeure d'intérêt du concept d'institution tient en effet à ceci, qu'il joint en lui, de façon synthétique, originaire et concrète, deux thèmes théoriques essentiels : 1) la référence à la question de l'événement: l'institution en effet est avant tout un événement et de l'événement elle possède certains caractères fondamentaux, à savoir la singularité, la facticité, la fécondité, l'anonymat. 2) $L a$ référence à l'ouverture d'un champ de reprises et de transformations ou, du point de vue historique, d'une tradition. Et un point essentiel consiste en ceci, que ces deux pôles doivent toujours être pensés ensemble, comme les deux faces d'un même phénomène enchevêtrées dans des relations multiples. C'est justement l'articulation explicite de ces relations multiples qui permet de montrer l'institution historique comme une voie pour penser l'événement historique. Il nous semble que l'on peut distinguer cinq aspects de la relation entre événement (institution au sens actif) et champ (institution au sens de « ce qui est institué »).

\footnotetext{
${ }^{1}$ Toutes les citations, RC (cours L'institution...), p. 60.

${ }^{2}$ IP, p. 37.
} 
1) L'événement de l'institution est un événement producteur de sens : il a tout son sens dans sa fécondité, donc dans le fait d'ouvrir une dimension, un champ d'effets et d'expérience, une tradition, qui vont au-delà de lui et qui co-constituent cependant son sens. Avec l'événement de l'institution, « le sens est déposé $[\ldots]$ comme à continuer, à achever sans que cette suite soit déterminée. L'institué changera mais ce changement même est appelé par sa Stiftung ${ }^{1}$. L'institution a un sens interne et $"$ ce sens interne induit justement sens externe parce qu'il est ouvert, qu'il est écart $»^{2}$. Merleau-Ponty exemplifie souvent cette dynamique par rapport aux produits culturels : dans le cas de la réalisation d'un livre, on découvre le projet qui l'anime seulement en l'écrivant, par ses réalisations partielles, où chaque vue partielle est en réalité une vue totale ; c'est pourquoi on peut dire qu' « un livre est une série d'institutions et manifeste que toute institution tend à la série $»^{3}$. Ainsi le peintre commence-t-il son œuvre toujours déjà placé dans un rapport avec ses œuvres précédentes et avec celles des autres peintres, rapport pour lequel d'une part le peintre entame son travail à partir d'une tradition déjà instituée, et pour lequel, d'autre part, c'est la tradition ellemême qui lui demande son prolongement, «comme si chaque pas fait exigeait et rendait possible un autre pas, comme si chaque expression réussie prescrivait à l'automate spirituel une autre tâche ou encore fondait une institution dont il n'aura jamais fini d'éprouver l'efficacité $\gg{ }^{4}$. Autant dire que l'événement ne vit que de ses reprises et transformations, qui le renouvellent et en même temps constituent son sens véritable.

2) En même temps le champ ainsi ouvert reste à son tour marqué par l'événement qui l'a institué et ne peut donc effacer la contingence de son avoir-eu-lieu-une-première-fois. Avec l'institution nous sommes en effet confrontés à un "lien de l'événement et de l'essence $»^{5}$. Ce que MerleauPonty affirme, à un moment donné de son cours, au sujet de l'histoire personnelle, est alors valable pour toute histoire : "L'idée d'institution est justement cela : fondement d'une histoire personnelle à travers la contingence $»^{6}$. Ce qui revient à dire qu'avec l'institution nous sommes face à un événement singulier et factuel qui instaure une dimension de sens, un événement empirique qui fonde une dimension «transcendantale ». Dans ce chiasme, le

\footnotetext{
${ }^{1} I d$.

${ }^{2}$ IP, p. 41.

${ }^{3} \mathrm{Id}$.

${ }^{4}$ LI, p. 85

${ }^{5}$ IP, p. 89.

${ }^{6}$ IP, p. 73.
} 
sens n'est pas une Idée intelligible donnée sub specie aeternitatis, mais une ouverture enracinée dans le monde concret et dans l'événement de son institution; la contingence pour sa part n'est pas un élément insignifiant ou un résidu de non-sens, puisqu'elle est plutôt caractérisée par sa fécondité. Précisément parce qu'il surgit dans un événement contingent, le sens d'une institution n'est pas une idéalité pleine et transparente, mais un sens " latéral » qui se donne par écart. C'est pourquoi « la culture ne nous donne donc jamais de significations absolument transparentes, la genèse du sens n'est jamais achevée $»^{1}$.

3) Si l'événement a son sens dans le champ qu'il ouvre, et si ce sens est sens par écart qui ne se donnera que dans ses reprises, il s'ensuit que chacune de ces reprises est une transformation ou, autrement dit, une nouvelle institution et donc un nouvel événement. À chaque fois il y a un nouvel événement qui modifie le champ institué dans lequel il s'insère et forme un nouveau champ institué avec son sens, selon une dynamique que dans le cours sur l'institution Merleau-Ponty exprime d'abord dans un langage plus immédiatement politique, même si c'est par des affirmations qui ont évidemment une validité générale : «Révolution et institution : la révolution est réinstitution, aboutissant à renversement d'institution précédente »; l'institution «n'est pas le contraire de [la] révolution: [la] révolution est une autre Stiftung ${ }^{2}$. Si l'on revient à l'exemple privilégié de la peinture, la tradition livre au peintre une tâche, mais, en accomplissant cette tâche, le peintre introduit dans la norme un écart personnel, qui s'institue comme nouvelle norme par rapport à laquelle d'autres écarts seront possibles : "Précisément parce qu'ils sont tous des moments de la peinture, chacun d'eux, s'il est conservé et transmis, modifie la situation de l'entreprise et exige que ceux qui viendront après lui soient justement autres que lui $»^{3}$.

4) Le mouvement en question inclut essentiellement la sédimentation et l'oubli de l'événement, sa cristallisation dans l' «institution» comme résultat, au sens de ce qui a été institué et occulte son origine événementielle, en fonctionnant du même coup comme ce qui «protège » de l'arrivée de nouveaux événements. Il s'agit d'un aspect qui nous semble représenter un moment structurel du mouvement en question: moins présent et valorisé dans les textes de Merleau-Ponty (ce qui n'est probablement pas fortuit, pour des raisons sur lesquelles nous ne pouvons pas nous arrêter ici), il n'est cependant pas complètement absent de ceux-ci, parce qu'ils posent au moins

\footnotetext{
${ }^{1}$ LI, p. 67.

${ }^{2}$ IP, respectivement p. 42 et p. 44.

${ }^{3}$ LI, p. 112 ; cf. aussi IP, p. 41.
} 
le problème du rapport entre la «lettre» et l'«esprit» de l'institution, la possibilité qu'une institution devienne lettre sans esprit ${ }^{1}$.

5) Les points précédents demandent en fait un approfondissement décisif du point de vue théorique, qui renvoie à une "stratification" dans l'analyse philosophique-phénoménologique du concept d'événement, au-delà aussi du cas spécifique de Merleau-Ponty ${ }^{2}$. La dynamique de l'institution implique en effet le problème du rapport entre un ordre général de l'événement, à savoir le caractère d'événement du sens en général irréductible à un fait ponctuel objectif, et les événements singuliers. Comme Merleau-Ponty le précise dans le cours de 1954-55, l'institution, en effet, n'est pas n'importe quel événement ponctuel, mais elle indique plutôt ces "événementsmatrices, ouvrant un champ historique qui a unité. L'institution [est] ce qui rend possible [une] série d'événements, [une] historicité : événementialité de principe $»^{3}$. Ces affirmations du cours font écho à des passages importants de Le langage indirect, où Merleau-Ponty distingue et lie à la fois les ordres de l'avènement et de l'événement : il faut « admettre l'ordre de la culture ou du sens comme un ordre original de l'avènement qui ne doit pas être dérivé de celui, s'il existe, des événements purs », contre une « histoire empirique qui n'est attentive qu'aux événements et reste aveugle aux avènements »; mais cela n'implique pas une pure séparation des deux plans, parce que «l'avènement est une promesse d'événements », l'ouverture du sens « exige [...] la succession $»^{4}$ et la série des événements pour pouvoir se déployer.

L'ensemble des analyses de Merleau-Ponty et la logique interne du concept d'institution esquissent donc un cadre global pour une compréhension de l'événement historique et de sa dynamique, ainsi que pour sa description phénoménologique. On peut voir aussi les analogies de ce concept avec le « retour » ou la « renaissance » de l'événement dans l'histo-

${ }^{1}$ Cf. IP, p. 43, 119, 122 ; nous rappellerons ensuite, d'un autre point de vue, un des passages en question.

${ }^{2}$ Les analyses de Merleau-Ponty nous permettent ainsi, à leur façon, de rejoindre une des préoccupations fondamentales de l'analyse de M. Zarader, qui, dans l'article déjà cité (« L'événement entre phénoménologie et histoire», art. cit., p. 290 sq.) souligne la coprésence (pas toujours thématisée explicitement) d'une double direction théorique dans les phénoménologies françaises de l'événement : l'analyse du phénomène de l'événement, donc de l'événement comme phénomène spécifique et singulier, avec ses traits caractéristiques; la pensée de l'événement au sens de l'événementialité générale de l'apparaître et de l'être lui-même, événementialité qui est la source et l'ouverture de tout phénomène.

${ }^{3}$ IP, p. 44.

${ }^{4}$ LI, respectivement p. 109, 99-100, 112. 
riographie des dernières décennies. Si, selon la formule de F. Dosse citée plus haut, il s'agit de considérer l'événement non plus en amont (pour en établir les causes), mais en aval (pour en analyser les traces et les métamorphoses), l'institution historique au sens merleaupontien montre une analogie remarquable avec ce concept d'événement et lui fournit un cadre théorique : l'institution historique est en effet un événement contingent dont le sens réside dans son ouverture, à savoir dans le champ d'expérience et dans la tradition qu'il fonde, dans la série de ses reprises qui seront autant de transformations et qui en constituent le sens. Dans ce cas aussi, on pourra affirmer que "l'événement est ce qu'il devient». La fécondité du concept merleaupontien d'institution et sa convergence avec les analyses de l'historiographie tiennent, entre autres, au fait que l'événement est envisagé ici dans le concret de ses relations avec la série de ses possibles répétitions et transformations, avec un champ d'expérience dans lequel il s'inscrit et qu'en même temps il modifie. Il s'agit d'un aspect qui distingue les analyses merleaupontiennes de celles des phénoménologies françaises contemporaines de l'événement.

Relevons encore trois points pour enrichir cette phénoménologie de l'institution historique en tant qu'elle implique une phénoménologie de l'événement historique.

Par sa nouveauté et par force d'ouverture, l'événement appelle à ses reprises de notre part, parmi lesquelles un rôle majeur est évidemment joué par la mémoire : l'événement compris par les historiens comme «trace signifiante » a en effet son corrélat dans un «tournant mémoriel ${ }^{1}$ dans l'historiographie, qui analyse et prolonge le rôle central de la mémoire dans le rapport de notre époque aux événements passés. Merleau-Ponty aussi, comme nous l'avons vu, lie l'institution à la capacité de donner au passé « une nouvelle vie» comme «forme noble de la mémoire ${ }^{2}$. Événement et mémoire sont ainsi dans un double rapport : l'événement fait appel à notre mémoire et la suscite, la mémoire à son tour donne à chaque fois une nouvelle vie à l'événement en le reprenant, ce qui veut dire du même coup en transformant et en réinstituant son sens.

${ }^{1}$ F. Dosse, La renaissance de l'événement, op. cit., p. 180 ; cf. plus en général p. 167-183, où F. Dosse souligne la diffusion de ce thème dans l'historiographie contemporaine et son lien avec la nouvelle conception de l'événement.

${ }^{2}$ Cf. D. Meacham, "The "Noble" and the "Hypocritical" Memory : Institution and Resistance in the Later Merleau-Ponty », Philosophy Today, n. 4, 2009, p. 233-243, texte auquel nous renvoyons aussi pour une analyse précise des rapports temporels de l'institution. 
Une pensée de l'événement, et de ses rapports avec la mémoire, implique évidemment aussi une certaine conception de la temporalité, laquelle se montre en fait comme un modèle structurant toute la dynamique de l'institution : «Le temps est le modèle même de l'institution : passivitéactivité, il continue, parce qu'il a été institué, il fuse, il ne peut pas cesser d'être, il est total parce qu'il est partiel, il est un champ ${ }^{1}$. La temporalité de l'événement n'est pas celle, chronologique, du temps objectif, puisque l'événement n'est pas un simple fait qui s'insère dans la série des instants ; elle ne se réduit pourtant pas non plus à une temporalité subjective au sens de la temporalité du vécu de l'événement. Il s'agit plutôt de ce que MerleauPonty appelle une "transtemporalité originaire ", qui est l' " institution à l'état naissant $»^{2}$ : dans l'événement de l'institution le présent appelle une poursuite dans l'avenir sans pourtant le nécessiter et répond à l'appel de ce qui est déjà institué en le reprenant, le passé se dépasse ouvrant la série de ses transformations et le futur est retour rétrospectif vers l'origine.

Remarquons enfin que, dans la section de son cours de 1954-55 dédiée à l'institution historique, Merleau-Ponty s'appuie sur un ouvrage de l'historien Lucien Febvre ${ }^{3}$ pour réfléchir sur l'opération de l'historien qui compare des époques différentes. D'un côté, la pensée n'a pas un accès absolu et direct à une autre époque ou à une autre civilisation, mais seulement «par l'autocritique de ses catégories, par pénétration latérale, et non par ubiquité de principe $»^{4}$. De l'autre côté, quand Merleau-Ponty veut résumer le "vrai sens de l'étude de Febvre », il affirme significativement que, s'il faut certes en retenir le plaidoyer contre l'anachronisme, il ne faut pourtant pas conclure par là à un "agnosticisme historique » et à l'idée de l' "insularité des temps", parce que, "s'ils étaient insulaires, nous ne verrions même pas leur différence ${ }^{5}$. Merleau-Ponty renvoie alors la possibilité de la confrontation historique justement aux notions d'institution et d'horizon: " En partant du tout, en interprétant les documents les uns par les autres, on réactive horizon $»^{6}$. Il s'agit donc de se laisser « entraîner au Nachvollzug par les documents ", effectuer une réactivation de ce qui nous est livré comme déjà fait pour réveiller les horizons institutionnels d'en-

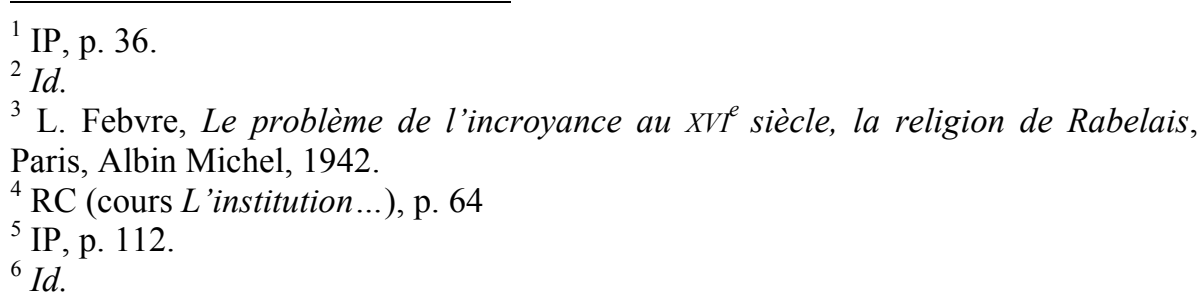


semble dont ces documents font partie. Ce qui implique deux aspects : d'une part, ces horizons étaient, dès leur institution, ouverts à leur propre changement et peuvent par conséquent être mis en communication avec notre horizon historique présent; d'autre part, réveiller les horizons institutionnels d'un document, d'une opinion, d'une position, signifie alors aller «audessous des opinions, les nôtres et les siennes, jusqu'au sol », pour révéler ainsi « une communication d'ordre existentiel, non notionnel». Et MerleauPonty de conclure : "En cela, l'institution de chaque temps, prise concrètement, comme horizon, est aussi moyen de comprendre les autres temps $»^{1}$. Nous verrons ensuite que ce qui émerge de cette analyse n'est que l'une des expressions d'une position "métaphysique » de fond qui parcourt plusieurs textes de Merleau-Ponty.

\section{L'institution : une pensée de l'historicité}

Tout ce que nous avons rappelé le montre déjà : si nous avons souligné jusqu'à maintenant surtout la possible interaction du concept d'institution de Merleau-Ponty avec une problématique strictement historique de l'événement, ses analyses ne se limitent pas à une description phénoménologique de l'événement historique. Toute une compréhension de l'historicité, dans sa portée "transcendantale » et " ontologique », peut en effet être dégagée de ces textes et surtout au début des années 1950 cette question semble en effet représenter une préoccupation majeure pour Merleau-Ponty, pour lequel l'histoire, comprise à partir de la logique de l'institution et de l'expression, pourrait redevenir «ce qu'elle doit être pour le philosophe : le centre de ses réflexions $[\ldots]$ comme le lieu de nos interrogations et de nos étonnements ${ }^{2}$.

\footnotetext{
${ }^{1}$ Toutes les citations, IP, p. 114 et note $a$ de la même page; nous soulignons « jusqu'au sol ». Relevons aussi que, en esquissant le programme de cette section du cours, Merleau-Ponty indique une possible confrontation avec l'œuvre de F. Braudel sur la Méditerranée, où la «longue durée » braudelienne aurait été interprétée à la lumière de la dynamique de l'institution: "Exemple d'institution historique i. e. donnant lieu à reprise et fonctionnant dans un horizon d'histoire universelle : la Méditerranée » (IP, p. 106). Cette confrontation ne sera cependant pas développée dans le cours. Pour quelques indications sur un usage " heuristique » des analyses de Merleau-Ponty dans le domaine de l'histoire, cf. N. Piqué, "Merleau-Ponty et l'histoire sauvage », Rue Descartes, n. 70, 2010, p. 74-87, en ligne à l'adresse : http://www.cairn.info/revue-rue-descartes-2010-4-page-74.htm

${ }^{2}$ LI, p. 113
} 
L'enjeu est représenté par une compréhension phénoménologique, à la fois concrète et non empirique, de l'historicité de notre expérience, de "l'histoire comme milieu de vie ${ }^{1}$ — compréhension phénoménologique qui cherchera aussi, de plus en plus, à expliciter les assises ontologiques de ses propres analyses. La logique de l'institution implique en effet une vision de l'historicité comme milieu qui joint de façon indissoluble la contingence et la fécondité, l'événement et la tradition, l'ancrage dans l'expérience par un événement singulier et l'ouverture d'un sens qui ne se réduit à aucun fait d'expérience, l'histoire déjà instituée et une histoire instituante en train de se faire. C'est justement par cet entrelacs de la contingence, de la fécondité, de la tradition, entrelacs résumé dans la figure de l'institution, que MerleauPonty parviendra à redéfinir le sens même de l'histoire : "Comment appeler, sinon histoire, ce milieu où une forme grevée de contingence ouvre soudain un cycle d'avenir, et le commande avec l'autorité de l'institué ? $»^{2}$.

Une telle vision de l'historicité implique le dépassement des limites et des dualismes des ontologies traditionnelles. Il s'agit d'abord de dépasser l'opposition entre une vision anhistorique et un empirisme qui réduirait l'histoire à une suite de faits dépourvue de sens : « Il n'y a pas histoire si le cours des choses est une série d'épisodes sans lien, ou s'il est un combat déjà gagné dans le ciel des idées. Il y a histoire s'il y a une logique dans la contingence $»^{3}$. C'est justement cette « logique dans la contingence » que MerleauPonty vise à déceler, puisqu'il s'agit de montrer, d'une part, que l'événement contingent ouvre le champ d'une tradition régie par un sens et que, d'autre part, ce sens ne se donne que dans le champ historique, concret et contingent, dans lequel il s'incarne. Plus précisément, un événement contingent s'intègre dans une tradition déjà instituée en assumant par là un sens, mais du même coup il modifie cette tradition, en introduisant un écart qui inaugure une nouvelle norme de sens : "Donc l'institution visible n'est que le support d'un esprit de l'institution, non certes immuable [...] mais dont le changement même est réaction de l'événement sur l'institué. Il faut de l'institué pour qu'il y ait registre ouvert, histoire $»^{4}$.

Ces dernières formulations nous permettent aussi de faire émerger la convergence entre les analyses consacrées au concept d'institution et la confrontation de Merleau-Ponty avec la linguistique saussurienne, qui a joué, comme on sait, un rôle capital dans le développement de sa pensée à partir de

\footnotetext{
${ }^{1} \mathrm{RC}$ (cours Matériaux pour une théorie de l'histoire), p. 45.

${ }^{2}$ M. Merleau-Ponty, « De Mauss à Claude Lévi-Strauss », in Signes, op. cit., p. 199.

${ }^{3} \mathrm{RC}$ (cours Matériaux pour une théorie de l'histoire), p. 46.

${ }^{4}$ IP, p. 40.
} 
la fin des années 1940. «Saussure pourrait bien avoir esquissé une nouvelle

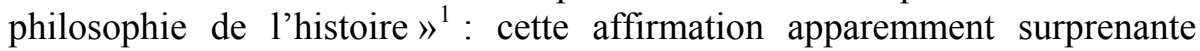
indique bien que les deux voies du concept d'institution et de la réflexion sur la linguistique ne sont que les deux aspects d'une même enquête, qui recherche un modèle de compréhension du sens historique. «La théorie du signe, telle que la linguistique l'élabore, implique peut-être une théorie du sens historique qui passe outre à l'alternative des choses et des consciences $»^{2}$ et dans laquelle on peut observer clairement «la présence de l'individu à l'institution et de l'institution à l'individu " ${ }^{3}$. Dans tout acte de parole, en effet, le sujet exprime sa volonté de parler, son intention et son style singulier, tout en étant en même temps tourné vers la communauté linguistique dont il fait partie et vers le système de la langue déjà instituée. Ce qui devient encore plus clair dans le cas d'un changement linguistique, puisque les sujets parlants introduisent un nouvel emploi des moyens linguistiques déjà subsistants et que ce nouvel emploi devient à son tour systématique en s'intégrant dans le tout de la langue :

Le fait contingent, repris par la volonté d'expression, devient un nouveau moyen d'expression qui prend sa place et a un sens dans l'histoire de cette langue. Il y a là une rationalité dans la contingence, une logique vécue, une autoconstitution dont nous avons précisément besoin pour comprendre en histoire l'union de la contingence et du sens ${ }^{4}$.

L'interprétation que Merleau-Ponty, toujours à partir du cadre saussurien, donne des rapports langue/parole, diachronie/synchronie, confirme ce parallèle. Les deux pôles de ces rapports ne peuvent en effet rester séparés. D'une part, "la synchronie enveloppe la diachronie", car, si à chaque moment, selon une coupe transversale, le langage est système, il faut qu'il le soit aussi

\footnotetext{
${ }^{1}$ M. Merleau-Ponty, Éloge de la philosophie et autres essais, Paris, Gallimard, collection «Folio», 1989, p. 56. Pour un commentaire de cette affirmation, cf. É. Bimbenet, Nature et humanité: le problème anthropologique dans l'œuvre de Merleau-Ponty, Paris, Vrin, 2004, p. 222 sq. Nous nous limitons ici à indiquer rapidement le parallèle avec les analyses sur la linguistique à l'intérieur de notre parcours, mais le thème devrait naturellement être l'objet d'une étude développée. Sur la convergence entre les réflexions sur l'histoire et sur la langue chez MerleauPonty, cf. les analyses éclairantes de J. Revel, Foucault avec Merleau-Ponty. Ontologie politique, présentisme et histoire, Paris, Vrin, 2015, p. 156 sq.

${ }^{2}$ M. Merleau-Ponty, Éloge de la philosophie et autres essais, op. cit., p. 56.

${ }^{3} \mathrm{Id}$.

${ }^{4}$ Id.
} 
dans son développement diachronique : les faits linguistiques contingents qui ont lieu dans l'histoire s'incorporent en effet dans un système du langage qui est, à chaque fois, doué d'une logique interne. D'autre part, «la diachronie enveloppe la synchronie » : si le langage, envisagé dans son histoire ou selon une coupe longitudinale, implique des hasards et l'avoir-lieu de nouveaux faits linguistiques contingents, " il faut que le système de la synchronie à chaque moment comporte des fissures où l'événement brut puisse venir s'insérer ${ }^{1}$. Le couple synchronie/diachronie implique évidemment aussi une certaine façon de comprendre l'histoire et l'enveloppement réciproque esquissé ici par Merleau-Ponty est analogue à celui que l'on peut relever, dans l'institution, entre l'événement et le champ : il y a un sens immanent dans le développement d'un champ d'expérience ou d'une tradition, car chaque événement contingent s'insère dans un champ déjà institué et doué de sa logique interne; le sens d'une institution, en même temps, est sens par écart, implique des fissures qui laissent advenir des nouveaux événements lesquels reconfigurent la situation donnée.

Dans le sillage de la problématique husserlienne, Merleau-Ponty voit dans l'institution aussi une mise en question de la séparation entre empirique et transcendantal, que l'on peut repérer en particulier dans un texte comme la célèbre Origine de la géométrie. Husserl cherche ici à penser la constitution des idéalités à partir d'une institution originaire et singulière dans l'histoire, ce qui implique, selon Merleau-Ponty, que «[pour] Husserl, finalement, l'origine transcendantale ne peut pas être autre que l'origine empirique : il y a dans l'origine empirique un intérieur, une histoire concevable à expliciter, et qui est là sous forme de champ $»^{2}$. Merleau-Ponty reviendra sur ce texte husserlien dans son cours de $1959^{3}$, où il lie de façon étroite le concept de Stiftung à celui d'horizon, dont il souligne à plusieurs reprises l'importance capitale. La Stiftung, en tant qu'instauration d'un sens qui se déploie dans le mouvement entre Urstiftung, Nachstiftung et Endstiftung, est l'ouverture des horizons dynamiques, pré-subjectifs et pré-objectifs de notre expérience : «Le rapport Vorstiftung-Nachstiftung = solution du problème passé-présent, moi-autrui, moi-mon futur ${ }^{4}$. Par l'ensemble de son interprétation, que nous

\footnotetext{
${ }^{1}$ Toutes les citations, M. Merleau-Ponty, « Sur la phénoménologie du langage », in Id. Signes, op. cit., p. 140.

${ }^{2}$ IP, p. 100.

${ }^{3}$ M. Merleau-Ponty, Notes de cours sur L'origine de la géométrie de Husserl, suivi de Recherches sur la phénoménologie de Merleau-Ponty, sous la direction de R. Barbaras, Paris, PUF, 1998. Le titre originaire du cours donné par Merleau-Ponty était Husserl aux limites de la phénoménologie.

${ }^{4}$ Ibid., p. 23.
} 
ne pouvons pas reconstruire ici, Merleau-Ponty est conduit aussi à indiquer un dépassement des limites des analyses husserliennes et une radicalisation du concept d'institution, qui vise surtout le statut de l'institution originaire et de la sédimentation :

Il n'y a sens, et particulièrement sens fécond, sens capable de fonder toujours et toujours de nouvelles acquisitions, que par sédimentation, trace [ici pousser Husserl : la Stiftung est non pas pensée enveloppante, mais pensée ouverte, non pas visée et Vorhabe du centre effectif, mais visée à côté qui sera rectifiée, non pas position d'une fin, mais position d'un style, non pas prise frontale, mais écart latéral, algue ramenée des profondeurs] ${ }^{1}$.

Si l'institution est écart, il faudra alors rendre à la sédimentation et à l'oubli leurs droits : il n'y a pas de formation du sens en dehors du processus de sédimentation d'une tradition et l'oubli du sens originaire n'est pas qu'un phénomène négatif, parce qu'il est ce qui permet d'avancer et de produire quelque chose de nouveau. Mais si la tradition est " oubli des origines », il s'ensuit qu'on ne saurait "récupérer la totalité de l'Urstiftung » et saisir le surgissement dans une «coïncidence absolue ${ }^{2}$. C'est pourquoi le concept de validité inconditionnée atemporelle de l'idéalité devient problématique. Mais tout cela «n'est pas un relativisme, un historicisme à cause de notion d'horizon $»^{3}$ : le passé n'est pas simplement rejeté comme faux, puisque, compris comme horizon et institution, il recelait déjà en soi le développement vers notre présent ; celui-ci à son tour rassemble en soi l'héritage du passé et l'ouverture à un avenir qui le dépassera sans le réfuter. "Mais ce n'est pas une $\varphi$ du savoir absolu à cause de même notion d'horizon ${ }^{4}{ }^{4}$ : il n'y a pas conservation et dépassement qui soient la même chose comme chez Hegel, mais une double circulation du passé vers l'avenir et de l'avenir vers le passé, circulation ouverte et instable, qui inclut aussi les moments de la sédimentation et de l'oubli.

La distinction déjà rappelée entre avènement et événement renvoie elle aussi au-delà du plan d'une phénoménologie de l'événement historique stricto sensu, pour soulever la question d'un sens événementiel généralisé qui ne se réduit à aucun événement particulier, voire la question d'un genre d'être qui régit l'expérience de l'histoire et échappe aux catégories de l'ontologie traditionnelle. Cette "événementialité de principe », exprimée

${ }^{1}$ Ibid., p. 30 .

${ }^{2}$ Ibid., p. 35.

${ }^{3}$ Ibid., p. 37.

${ }^{4}$ Id. 
aussi par l'idée de l'institution comme " événement-matrice », prépare donc l'espace pour les concepts d' "histoire sauvage» et "histoire verticale » auxquels Merleau-Ponty aura recours de plus en plus par la suite. Il est remarquable que l'expression « histoire sauvage » soit utilisée déjà dans le cours sur la passivité, donné par Merleau-Ponty pendant la même année que celui sur l'institution, dans un passage qui souligne comment l'historicité se situe en deçà de l'opposition entre une activité du sujet et une passivité des choses :

Ici aussi la genèse a double sens : du donné à nous, et aussi de nous au donné [...] Ou plutôt, non pas deux mouvements contraires [...] ce qui est donné c'est leur croisement, l'articulation l'une sur l'autre des perspectives. La " vérité » du passé [à concevoir] ni en soi comme si je le survolais, ni pour mon présent seulement [...] la vérité, comme ce qui juge et le passé vécu par les hommes du passé et mon entreprise : comme leur appartenance à une seule histoire. Donc réveiller l'histoire sauvage (par delà l'histoire " objective", qui ne s'occupe pas des consciences, et par delà l'histoire comme appendice de mon aventure personnelle) [...] nous, comme les hommes du passé, n'avons que des significations et des situations ouvertes dont le sens est en genèse ${ }^{1}$.

L'histoire sauvage est donc l'expression d'un sens en genèse qui permet le croisement entre mon présent et le passé et qui se situe en deçà d'une histoire objective et d'une histoire subjective : c'est le mouvement qui fait que "même l'histoire "objective" ne vit que dans notre vie, et même nos Sinngebung s'appuient sur configuration du passé, sont urgestiftet en lui $»^{2}$. L'histoire a lieu toujours à partir d'un champ déjà institué, qui est formé par la sédimentation de déterminations déjà données et dans lequel le sujet est situé, mais ce champ est à son tour dynamique, il est appel à son propre développement dans les reprises des sujets ${ }^{3}$. Ce qu'il s'agit de penser alors est le mouvement d'une histoire instituée/instituante, sans la présupposition d'une subjectivé constituante, mais comme chiasme du poids des choses et de nos actions.

\footnotetext{
${ }^{1}$ M. Merleau-Ponty, Le problème de la passivité: le sommeil, l'inconscient, la mémoire, in IP, p. 178-179. ${ }^{2} I d$.

${ }^{3}$ Cf. J. Revel, Foucault avec Merleau-Ponty, op. cit., p. 153 sq., 179 sq., qui insiste efficacement sur l'effort de Merleau-Ponty de penser conjointement l'histoire déjà faite (l'histoire sédimentée et déjà instituée dans laquelle nous sommes placés) et l'histoire se faisant (l'ouverture d'une différence possible et d'une nouveauté).
}

\section{9}


L'expression «histoire sauvage » utilisée ici anticipe ainsi de façon singulière l'usage fréquent de la formule «Être sauvage » (ou parfois « esprit sauvage ») dans Le visible et l'invisible, où le qualificatif "sauvage" est souvent accompagné par celui de «vertical $»^{1}$. Dans ce réseau de concepts on peut alors comprendre l'apparition dans ce dernier texte aussi d'une « histoire verticale $»^{2}$. La verticalité de cette histoire tient au fait qu'il s'agit de penser la "présence" sui generis d'un sens qui, en deçà du déploiement horizontal/chronologique de l'histoire, insiste verticalement dans chaque moment historique, en lui donnant à la fois sa profondeur, son épaisseur et son ouverture aux autres moments historiques. Il y a donc une ouverture de chaque moment historique à la transcendance d'un même Être vertical ou sauvage, comme Merleau-Ponty l'exemplifie surtout à propos de l'histoire de la philosophie :

montrer qu'il y a transcendance, certes, entre les philosophies, pas de réduction à un plan unique, mais que, dans cet échelonnement en profondeur, elles renvoient quand même l'une à l'autre, il s'agit quand même du même Être Montrer entre les philosophies rapport perceptif ou de transcendance. Donc histoire verticale, qui a ses droits à côté de l'histoire de la philosophie «objective $»^{3}$.

\section{Une « métaphysique de l'histoire»}

Si Le visible et l'invisible développera les implications ontologiques des analyses précédentes, Merleau-Ponty n'aura pourtant pas attendu 1959/1960 pour pousser au plus haut niveau l'ambition de ses recherches sur ces thèmes, en formulant le projet d'une "métaphysique de l'histoire », comme on peut le lire dans le résumé du cours sur l'institution, qui se termine en affirmant : «C'est ce développement de la phénoménologie en métaphysique de l'histoire que l'on voulait ici préparer ${ }^{4}$. La formule peut paraitre surprenante et énigmatique, d'autant plus qu'elle n'apparaît quasiment pas dans le texte du cours. On peut essayer de l'expliquer dans quatre directions.

\footnotetext{
${ }^{1}$ Cf. M. Merleau-Ponty, Le visible et l'invisible, suivi de Notes de travail, texte établi par C. Lefort, Paris, Gallimard, 1964, p. 228, 229, 251, 253, 254 ; dorénavant cité comme VI.

${ }^{2}$ VI, p. 234, 237, 250, 272.

${ }^{3}$ VI, p. 237.

${ }^{4} \mathrm{RC}$ (cours L'institution...), p. 65.
} 
En premier lieu, les lignes immédiatement précédentes du résumé indiquent le contexte anti-hégélien de cette affirmation : l'hégélianisme est, en un sens, "la découverte de la phénoménologie », c'est-à-dire de la liaison vivante entre les éléments du monde et de la manifestation de l'être dans l'histoire, mais il la subordonne à la vision systématique et atemporelle du savoir absolu ; si, par contre, la phénoménologie n'est pas seulement une introduction au savoir vrai, mais «elle demeure tout entière dans la philosophie », elle doit prendre à son compte «la méditation de l'être » ${ }^{1}$ pour s'élever elle-même à un niveau métaphysique, ou, en d'autres termes, elle doit élaborer une conception de l'être correspondante qui justifie l'impossibilité de son dépassement et par là la critique à Hegel.

Cela requiert évidemment, en deuxième lieu, une profonde révision de la structuration hégélienne du cours des figures historiques, comme on peut le lire dans un autre passage du cours. Après avoir affirmé que l'institution implique à chaque fois un certain oubli et l'avancement vers le nouveau, l'ouverture d'un avenir qui n'est pas nécessité et une reprise du passé qui le laisse pourtant dans son originalité, Merleau-Ponty affirme :

Dans cette intériorité avec échappement, ce passage de décentration à décentration, sans décentration absolue, dans cette extériorité qui n'empêche pas les enjambements, il y a vraiment union de l'extériorité et de l'intériorité, à chaque moment, tandis que Hegel ne les unifie qu'en les poussant à l'absolu ${ }^{2}$.

Ce que l'institution nous donne à penser, c'est donc l'union dans chaque moment (on pourrait dire, dans chaque figure) de l'intériorité et de l'extériorité, de l'universel et du particulier, union qui n'est pas une synthèse finale dans un savoir absolu et laisse donc ouverte la possibilité d'écarts, d'enjambements, de nouvelles décentrations. Cette forme d'union - à l'aide de l'idée de "partie totale», selon l'expression que Merleau-Ponty utilisera fréquemment dans d'autres textes - permet de réaliser une " autonomisation » des figures historiques sans en opérer une relève dans le savoir absolu et de penser ainsi une «métaphysique de l'histoire».

En troisième lieu, la formule " métaphysique de l'histoire » n'est utilisée, à notre connaissance, qu'une seule fois dans le cours, et ce dans une note ajoutée par Merleau-Ponty à son texte principal, à la fin de laquelle il écrit : «Problème de la métaphysique de l'histoire : comment la médiation des rapports personnels par les choses est-elle possible ? statut de la Vernunft par

\footnotetext{
${ }^{1} I d$.

${ }^{2}$ IP, p. 100.
} 
rapport à la "Nature" " ${ }^{1}$. La question posée n'est pas suivie d'une explication de la part de Merleau-Ponty. On peut relever qu'elle fait écho à la page précédente du cours où Merleau-Ponty, indiquant le passage de l'institution individuelle à l'institution intersubjective et historique, reprend la définition marxiste du capital: "[Idée d'une] rationalité originaire dans [1']histoire privée: l'Histoire [devient] "relation entre personnes médiatisée par des choses" $\gg{ }^{2}$. Ne pouvant développer tous les possibles renvois de ces affirmations, en l'absence aussi d'une indication explicite de la part de MerleauPonty dans cette note, nous pouvons en tout cas relever que le problème de la «médiation des rapports personnels par les choses » peut être lié au passage que nous avons cité à propos de l'histoire sauvage : celle-ci est en effet le milieu originaire qui se situe en deçà de l'alternative entre l'histoire objective et l'histoire subjective, entre la passivité et l'activité, et qui permet donc d'en penser la «médiatisation» originaire. On serait donc ici renvoyés à la tâche de remonter à ce sol ontologique commun aux personnes et aux choses, qui rend possible leur communication et interaction dans une "histoire sauvage $»^{3}$.

En quatrième lieu, on peut éclaircir la référence à une « métaphysique de l'histoire» par un autre texte, qui renvoie également à une "métaphysique » à partir des questions de l'histoire et du rapport entre les différentes humanités. Il s'agit des dernières denses pages, qu'il faudrait commenter dans le détail, du programme de travail rédigé par Merleau-Ponty en 1951 pour sa candidature au Collège de France ${ }^{4}$. Dans le cadre d'une interprétation de l'histoire comme symbolisme, en parallèle aux thèmes du langage et de l'expression, Merleau-Ponty revendique la possibilité de parler d' «une histoire de l'humanité » ou d' "une humanité », et il affirme :

L'idée d'une histoire unique ou d'une logique de l'histoire est, en un sens, impliquée dans le moindre échange humain [...] l'anthropologie suppose

\footnotetext{
${ }^{1}$ IP, p. 48, n.

2 IP, p. 47.

${ }^{3}$ Ce qui nous semble confirmé par ce passage de la Préface à Signes, op. cit., p. 36 : «À quoi bon se demander si l'histoire est faite par les hommes ou par les choses, puisque de toute évidence les initiatives humaines n'annulent pas le poids des choses et que la "force des choses" opère toujours à travers des hommes ? [...] Il n'y a pas d'analyse qui soit dernière parce qu'il y a une chair de l'histoire ».

${ }^{4}$ M. Merleau-Ponty, «Un inédit de Maurice Merleau-Ponty », in Id., Parcours deux, 1951-1961, Verdier, Lagrasse, 2000, p. 36-48.
} 
toujours qu'une civilisation, même très différente de la nôtre, est à la limite compréhensible pour nous ${ }^{1}$.

Merleau-Ponty tient assurément à distinguer ce " rationalisme méthodique » d'un « rationalisme dogmatique » de type idéaliste ou matérialiste. Il n'en demeure pas moins qu'il évoque "une histoire totale - un seul tissu qui réunit toutes les entreprises de civilisations simultanées et successives $»^{2}$. Qu'est-ce qui fait ce rapport de sens, ce tissu commun? La pensée permanente et concordante de la pluralité des êtres humaines qui se reconnaissent comme semblables, pensée qui doit conduire aux problèmes d'un «homme transcendantal», d'une " "lumière naturelle" commune à tous ", d'un Logos qui transparaît à travers le mouvement de l'histoire : « Nous rejoignons ici les questions classiques de la métaphysique ${ }^{3}$. Le programme se termine en affirmant que le phénomène de l'expression permet d'envisager une spontanéité qui réunit la finitude et l'universalité, l'intériorité et l'extériorité, «qui accomplit ce qui paraissait impossible, à considérer les éléments séparés, qui réunit en un seul tissu la pluralité des monades, le passé et le présent, la nature et la culture ». Et Merleau-Ponty de conclure : «La constatation de cette merveille serait la métaphysique même » ${ }^{4}$. La méditation et l'expression de l'union en un seul tissu des monades, des civilisations et des époques - en d'autres termes, l'esquisse d'une « histoire totale» - serait donc " la métaphysique même » : par un tel programme ces pages contribuent à éclaircir la référence à une " métaphysique de l'histoire » du cours de 1954-55 et esquissent déjà celle qui ne cessera d'être la position merleaupontienne dans les textes des années suivantes.

En effet, comme nous l'avons vu, dans le cours sur l'institution, la discussion de l'ouvrage de l'historien L. Febvre permet à Merleau-Ponty d'affirmer la possibilité de principe d'une compréhension entre les époques, contre l'idée de leur insularité : l'institution d'un temps historique est aussi moyen pour comprendre les autres temps. Ajoutons que l'institution en général est définie aussi comme « la conséquence et la garantie de notre appartenance à un même monde $»^{5}$. Cette analyse est suivie alors par une confrontation avec Lévi-Strauss, dans laquelle Merleau-Ponty vise à critiquer le relativisme absolu de l'anthropologue par deux arguments. En premier lieu, la

${ }^{1}$ Ibid., p. 46.

${ }^{2}$ Ibid., p. 47, nous soulignons.

${ }^{3} I d$.

${ }^{4}$ Ibid., p. 48.

${ }^{5} \mathrm{RC}$ (cours L'institution...), p. 60. 
constatation de la relativité et de l'opacité de toutes les cultures est tributaire d'un regard de survol, d'un Kosmotheoros situé dans une position d'extériorité, si bien que la position de Lévi-Strauss est une conjonction, qui n'est paradoxale qu'en apparence, de relativisme absolu et savoir absolu ${ }^{1}$; à quoi il faudra opposer que le philosophe, au lieu d'être Kosmotheoros, « devient au contraire et justement [celui pour qui il y a] reconnaissance des particularités qui unissent $»^{2}$. En deuxième lieu, ce relativisme se contredit au moment où il s'énonce, parce qu'il prouve par là la possibilité pour une civilisation de parvenir à cette constatation universelle, ou, autrement dit, il méconnaît l'historicité particulière de la civilisation qui se pose la question du rapport aux autres humanités ${ }^{3}$. C'est dans le sillage de cette confrontation que Merleau-Ponty peut alors indiquer l'horizon dernier de son cours, même si c'est sous la forme d'une question ouverte, et tracer une distinction entre sociétés qui a son critère justement dans l'ouverture à l'universel :

Problème : y a-t-il un champ de l'histoire mondiale ou universelle ? Y a-t-il un accomplissement de visée? Une fermeture sur elle-même? Une société vraie ? La question reste [...] interrogation de l'histoire. Mais [il s'agit d'un] universel d'existence, non conceptuel, introduit par cette interrogation même. Il y a sinon une société vraie [...] du moins des sociétés qui se posent la question de la société vraie [...] [concevant l']idée d'une récupération de l'histoire par soi. Et il y a d'autres sociétés qu'on peut appeler fausses relativement à celles-là. Ce qui ne veut pas dire que sous certains rapports elles ne soient pas plus belles. Mais elles ne jouent pas le jeu mystérieux qui est de mettre tous les hommes dans le jeu, d'essayer le brassage vraiment universel, elles sont institutions selon la lettre et non selon l'esprit de l'institution, qui n'est pas de limiter, de prohiber, d'enfermer dans un îlot de coutumes, mais de mettre en route un travail historique illimité ${ }^{4}$.

Cette position se prolongera, en se transformant, dans les derniers textes, où l'appartenance des hommes et des époques à un même monde et à une seule histoire sera consacrée par l'idée d'une appartenance ontologique à la même «chair du monde ${ }^{5}$, qui constitue évidemment ce «seul tissu» que

\footnotetext{
${ }^{1}$ IP, p. 116-117, 120.

${ }^{2}$ IP, p. 108.

${ }^{3}$ Cf. IP, p. $44-45,107-108$.

${ }^{4}$ IP, p. 118-119, nous soulignons dans la dernière phrase ; cf. le passage analogue à la fin du cours, p. 122.

${ }^{5}$ Comme E. Bimbenet, Nature et humanité, op. cit., p. 292-296, le remarque, chez le dernier Merleau-Ponty c'est la chair, et non pas le langage, qui constitue le fait primordial et le sol qui garantit la possibilité d'une raison universelle.
} 
Merleau-Ponty évoquait dès son programme de 1951. Il est significatif en effet que le cours sur l'Origine de la géométrie (contemporain de la rédaction de Le visible et l'invisible) se termine par une analyse du manuscrit de Husserl sur le renversement de la doctrine copernicienne et en particulier de la notion de Terre : l'analyse de l'institution historique débouche enfin dans le thème du « sol » pré-objectif et " sauvage » de notre expérience et la Terre est le nom dans l'impensé husserlien de la « chair du monde ». La Terre est aussi le sol d'une Urhistorie, d'une histoire originaire dont Merleau-Ponty dit qu'elle « relie toutes les sociétés réelles ou possibles en tant qu'elles habitent toutes le même espace "terrestre" au sens large ${ }^{1}$. Il ne reste qu'à rappeler que Le visible et l'invisible confirme par le même geste le refus de "la finitude au sens empirique » et le fait d'être alors «pour la métaphysique $»^{2}$.

\section{Perspectives critiques : unité/différence, continuité/nouveauté}

Mais précisément ces analyses que nous avons rassemblées autour de la référence à une "métaphysique de l'histoire » nous semblent appeler des questions critiques, que nous concentrerons autour de deux thèmes: les couples unité/différence et continuité/nouveauté.

Nous l'avons vu: Merleau-Ponty affirme la possibilité de principe d'une compréhension des différentes époques et civilisations, en s'opposant aussi bien à un "agnosticisme historique " qu'au relativisme anthropologique absolu de Lévi-Strauss. Cette possibilité conduit même à l'idée d'une histoire unique et totale de l'humanité, d'un «seul tissu » qui lie la pluralité des monades aussi dans leurs différences historiques; et ce « seul tissu» aura enfin sa formulation et sa garantie ontologique dans les concepts de « sol» et de « chair » des derniers textes.

${ }^{1} \mathrm{RC}$ (cours Husserl aux limites de la phénoménologie), p. 170. Cf. aussi M. Merleau-Ponty, Notes de cours sur L'origine de la géométrie de Husserl, op. cit., p. 89 : «Que l'histoire ait un sens, qu'elle soit une, qu'elle soit dimension, cela : cette rationalité, est formé sur le rapport pré-objectif des hommes aux hommes et à la terre $»$.

${ }^{2}$ Plusieurs notes de travail de Le visible et l'invisible opposent l'infini objectif, positif, et l'ouverture inépuisable de l'invisible qui doit être pensée comme l'Offenheit du monde de la vie. Dans un de ces passages Merleau-Ponty écrit : "Je suis contre la finitude au sens empirique, existence de fait qui a des limites, et c'est pourquoi je suis pour la métaphysique. Mais elle n'est pas plus dans l'infini que dans la finitude de fait » (VI, p. 300). 
Malgré l'importance de la critique du relativisme, surtout en vertu de son principe phénoménologique, l'hypothèse d'une « histoire totale » est-elle vraiment tenable ? Et, si elle l'est, doit-elle nécessairement s'appuyer sur la présupposition ontologique forte de ce «seul tissu» représenté par la chair du monde ? Face à la multiplicité des civilisations, de leurs temporalités et de leurs historicités, ne risque-t-elle pas d'être encore une expression d'un regard très «occidental »? Du moins, dans la formulation que lui donne Merleau-Ponty, n'implique-t-elle pas des conséquences problématiques ? En effet, la garantie de la possibilité de l'interaction entre les cultures, contre un enferment historiciste et relativiste, semble, dans les textes de MerleauPonty, conduire à une subordination nette de l'altérité de ces cultures au profit de ce qui les relie, à une subordination de la différence en faveur de l'unité, de la continuité, de la possible universalité. La distinction entre les sociétés qui se posent le problème de l'ouverture universelle et les sociétés « fausses » par rapport à celles-ci, entre l'esprit et la lettre de l'institution, est l'expression à la fois la plus séduisante et la plus révélatrice de cette position. D'ailleurs, en abordant le problème de la communication entre les temps historiques, Merleau-Ponty affirme clairement: «En un sens la première condition est de savoir aussi qu'ils ne sont pas de part en part autres $»^{1}$.

Mais le risque qui parcourt ces pages est alors celui de considérer les différences, les discontinuités, les incompréhensions et les malentendus, bref toutes les expressions d'une altérité radicale, comme des «accidents » par rapport au vrai sol ontologique, comme des obstacles qu'il faut dépasser vers la vraie communauté (le «pour autrui vrai $»^{2}$ ) corrélative de l'histoire totale, au lieu de les assumer comme des éléments aussi constitutifs de l'historicité que ceux soulignés par Merleau-Ponty. En un sens on pourrait réitérer ici, mutatis mutandis, la querelle entre Derrida et Gadamer, et adresser aussi à Merleau-Ponty la question de savoir si ce qui est premier c'est le continuum de la compréhension ou bien plutôt «un certain rapport d'interruption », voire «l'interruption du rapport $»^{3}$ : non pas un enfermement historiciste, mais une interruption qui, comme expression de l'altérité, garde le rapport irréductiblement ouvert.

D'autre part, une « histoire sauvage » ou un « esprit sauvage», précisément en tant que «sauvages», ne devraient-ils pas impliquer aussi une

\footnotetext{
${ }^{1} \mathrm{IP}$, p. 114 , note $a$.

2 IP, p. 114.

3 Cf. J. Derrida, «Bonnes volontés de puissance (une réponse à Hans-Georg Gadamer) », Revue internationale de philosophie, n. 151, 1984, p. 343.
} 
certaine an-archie et une multiplicité irréductible ${ }^{1}$ ? Il faudrait alors se demander s'il n'y a pas lieu de faire place à une altérité et à une différence radicale d'une façon autre que celle relativiste, pour ainsi dire au-delà de l'alternative entre les positions de Merleau-Ponty et Lévi-Strauss. Ce qui demanderait avant tout de mettre en question les valeurs de continuité et unité qui commandent en profondeur toutes les analyses merleaupontiennes, et par conséquent le sol ontologique qui viendra cautionner ces valeurs dans Le visible et l'invisible.

C'est ce même sol ontologique - avec ce qui l'a préparé et ce qui en découle - qui implique des conséquences problématiques aussi au sujet de la deuxième question que nous voudrions soulever. Nous avons essayé de montrer, dans la première partie de notre texte, la fécondité du concept merleaupontien d'institution historique, sa possible contribution à une phénoménologie de l'événement historique, sa convergence avec certaines interprétations du « retour de l'événement » en historiographie. Comme nous l'avions remarqué, cette convergence et cette fécondité tiennent, entre autres, au fait que l'événement est envisagé ici dans le concret de ses relations avec la série de ses répétitions/transformations et avec un champ d'expérience dans lequel il s'inscrit. Il n'en demeure pas moins que cet aspect ne représente que l'un des éléments fondamentaux du concept d'événement historique, lequel en implique au moins un autre autant essentiel que le premier : l'événement comme irruption du nouveau, avoir-lieu d'une nouveauté radicale et imprévue qui contredit notre horizon d'attente, fait basculer nos catégories et, comme événement traumatique, laisse une «blessure » dans notre champ d'expérience. C'est aussi en vertu de cet aspect que l'événement demeure essentiel pour une compréhension de l'historicité et qu'il s'est réimposé comme incontournable pour l'historiographie, surtout pour le caractère fort "événementiel» de l'histoire contemporaine. Or le cadre conceptuel élaboré par Merleau-Ponty rencontrerait, nous semble-t-il, des difficultés pour rendre compte de l'irruption de la nouveauté historique et semble parfois même en délimiter par principe la portée ${ }^{2}$.

\footnotetext{
${ }^{1}$ La question est posée par M. Richir, «Communauté, société et Histoire chez le dernier Merleau-Ponty ", in M. Richir \& E. Tassin (éd.), Merleau-Ponty : Phénoménologie et expériences, Grenoble, Millon, 1992, p. 7-25, en particulier p. 19-20, qui, par un parcours différent, souligne également le primat problématique d'une certaine unité chez le dernier Merleau-Ponty et sa tension avec l'idée d'un « esprit sauvage». ${ }^{2}$ Cf. par exemple RC (cours Matériaux pour une théorie de l'histoire), p. 45 : «Il y aurait illusion prospective à faire cesser le présent au seuil d'un avenir vide, comme si chaque présent ne se prolongeait pas vers un horizon d'avenir $[\ldots] »$.
} 
L'insistance sur le fait que l'expression et l'institution ouvrent une histoire unitaire, que les différents peintres et les différentes œuvres d'un peintre sont comme les épisodes d' " une seule histoire " ${ }^{1}$ ou les réponses à «une seule tâche »" l'enracinement de l'historicité dans un même Être sauvage ou vertical, par conséquent l'idée selon laquelle les différentes figures historiques ne seraient que les expressions de cet Être (qui est, bien sûr, non positif et inépuisable) : tous ces éléments font tomber l'accent sur l'entre-appartenance des moments historiques, sur la fécondité du champ historique inauguré par l'événement, et on voit mal par contre quel pourrait être dans ce cadre théorique le statut de l'événement comme irruption du nouveau. C'est ce que plusieurs affirmations de Le visible et l'invisible confirment : le monde perceptif amorphe « ne contient aucun mode d'expression » et " pourtant les appelle et les exige tous », ce qui conduit à penser une « création qui est en même temps réintégration de l'Être ». Il faut rappeler aussi, et peut-être surtout, le passage que nous avons déjà cité à propos d'une « histoire verticale » de la philosophie : s'il n'y a « pas de réduction à un plan unique » des différentes philosophies, il faut cependant montrer que « dans cet échelonnement en profondeur, elles [les philosophies] renvoient quand même l'une à l'autre, il s'agit quand même du même Être - Montrer entre les philosophies rapport perceptif ou de transcendance $\gg{ }^{3}$. Comme on l'a justement remarqué, la pensée de Merleau-Ponty semble se mouvoir ici dans une tension constitutive : "penser la permanence d'un fond, tout en pensant le caractère inépuisable de ses expressions successives; poser son caractère englobant tout en affirmant l'impossibilité de toute coïncidence ou saisie définitivement adéquate ${ }^{4}$. Cette difficulté à rendre compte de la nouveauté de l'événement se lie probablement à une tendance plus profonde: la contingence constitutive de l'événement de l'institution, bien que soulignée par Merleau-Ponty, semble être le plus souvent reprise ou réabsorbée par le mouvement du sens qui se développe à travers elle et qui la dépasse ${ }^{5}$. En

\footnotetext{
${ }^{1}$ Cf. LI, p. 111 et 113 ; sur l'idée d'une histoire unique cf. aussi les passages déjà cités de IP, p. 179, et de «Un inédit de Maurice Merleau-Ponty », art. cit., p. 46-48. ${ }^{2}$ Cf. LI, p. 96 et 112.

${ }^{3}$ VI, respectivement p. 221, 247, 237.

${ }^{4}$ N. Piqué, «M. Merleau-Ponty et l'histoire sauvage », art. cit., p. 85.

${ }^{5}$ Cf. par exemple IP, p. 177. M. Carbone, «Amour et musique : thème et variations », Alter, n. 15, 2007, p. 119, remarque justement que dans le cours sur l'institution Merleau-Ponty ne pense pas encore de façon assez radicale l'élément d'expropriation impliqué par les thèmes de l'événement et de la contingence, dont le rôle est plutôt décrit en termes de réorientation du sens; l'auteur donne par contre une interprétation différente du développement suivant du parcours de Merleau-Ponty.
} 
d'autres termes, Merleau-Ponty semble négliger ce qui de la contingence fait essentiellement résistance à une appropriation dans et par le sens.

D'une part et d'autre, il s'agirait alors peut-être d'assumer toutes les conséquences de la pensée de l'institution, y compris ses conséquences « ontologiques» et déconstructrices de l'ontologie traditionnelle, sans pour cela la faire déboucher dans une "ontologie» et l'appuyer sur un Être charnel, qui en donnerait le sol d'une façon problématiquement unitaire et continue. Assumer toutes ces conséquences signifierait alors peut-être penser la contingence de l'événement aussi dans ce qu'elle a d'irréductiblement nouveau, inappropriable et expropriateur - et les séries des institutions dans ce qu'elles ont de radicalement différent et multiple ${ }^{1}$.

\footnotetext{
${ }^{1}$ Cet article a été rédigé dans le cadre du projet de recherche PHENINST, financé par une bourse "Marie Curie» de l'Union Européenne et réalisé auprès des Archives Husserl de Paris (ENS/CNRS).
} 